Background and aims A reduction in diagnostic errors is key to patient safety. Paediatric consultants and trainees were surveyed to elicit their perceptions regarding the frequency, contributing factors, and preventative strategies of diagnostic error.

Methods This online survey was pre-tested and administered using SurveyMonkey. Participants were invited to participate by email. Weighted averages of ranked outcomes were computed. Friedman's test was used to assess non-randomness of ranking.

Results The overall response rate was 38\% $(\mathrm{n}=310)$. Respondents included paediatric consultants (31.6\%) and trainees (65.9\%). 50\% of Consultants reported making a diagnostic error at least 1-2 times per quarter, this frequency was significantly higher among trainees (75.9\%) $(\mathrm{p}=0.027)$. 36.4\% and $29.7 \%$ of trainees and consultants respectively reported making a diagnostic error that results in patient harm at least once or twice per year $(\mathrm{p}=0.69)$.

Inadequate staffing levels and/or inexperience of healthcare staff was the most commonly reported system-related factor contributing to diagnostic error. Inadequate data gathering and failing to consider other possible diagnoses were the most common causes of cognitive process breakdown. Excessive workload and physician fatigue were highly ranked additional factors. With regard to reducing diagnostic error, asking for second opinions and increased access to consultants were ranked as the most effective strategies to reduce diagnostic error.

Conclusion This study highlights diagnostic error as a potentially under-recognised patient safety issue. A few key systemicand cognitive-related factors are identified, while many factors contribute equally to diagnostic error. Further research should focus on methods to instruct clinicians on strategies to reduce recurrence.

\section{PS-121 IMPROVING THE DELIVERY OF NEONATAL RESEARCH - DEVELOPING AN INFORMATION LEAFLET FOR PARENTS}

V Oliveira, E Reus. Paediatric Research, Evelina London Children's Hospital, London, UK

\subsection{6/archdischild-2014-307384.416}

Background Medical and technological developments within Neonatal Care over the last decade have increased the demand for Neonatal Research. ELCH's Neonatal Unit is highly research
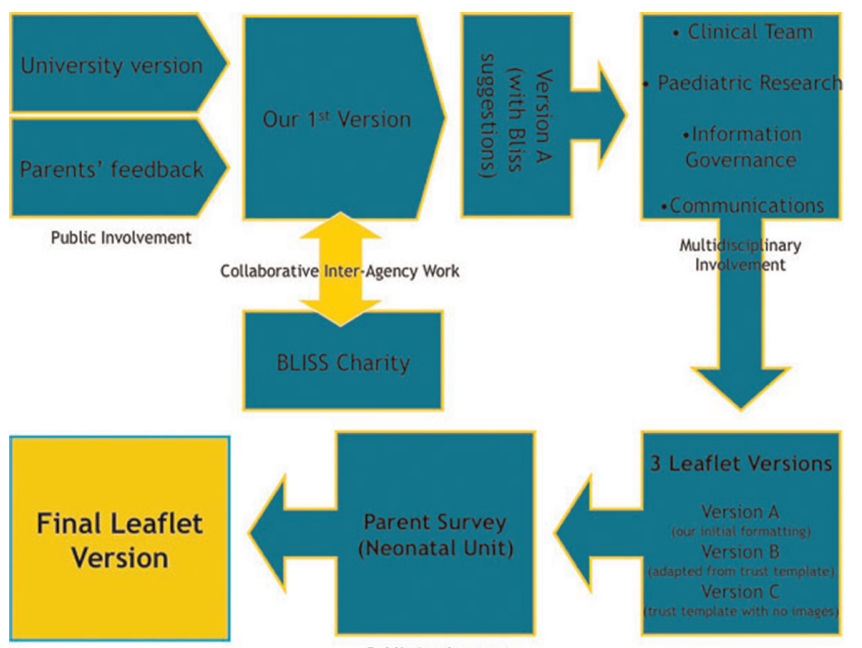

Public Involvement

Abstract PS-121 Figure 1 Key topics of information valued by parents

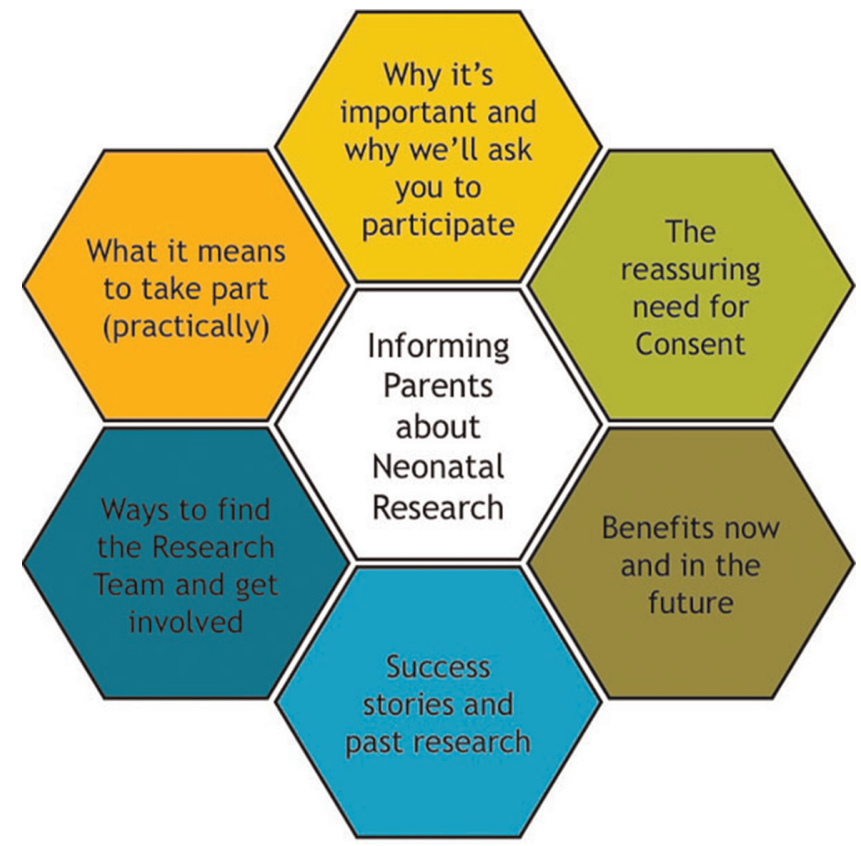

Abstract PS-121 Figure 2 Key topics of information valued by parents

active. Parents can be approached for up to 5 or 6 studies. As a consequence, we observed an increased need for a sensitive management of multi-study approaches.

Aims As part of a service improvement project to improve the delivery of Neonatal Research, we aimed to develop an information leaflet for parents of infants admitted to the Neonatal Unit. Methods We invited parents to help construct the leaflet involving them in all stages of development of the leaflet and worked closely to ensure multidisciplinary input. We did a survey asking parents to elect their favourite version of the leaflet from three different layouts.

Results All parents found the leaflet helpful $(\mathrm{n}=18)$. The preferred version was mostly described as "clear" and "informative". Parents selected pictures that they would like to see in the leaflet and considered them helpful. The topics they valued the most are represented in Figure 1.

Conclusions Parental involvement in all stages of research can present many opportunities for service improvement. Enhanced recruitment rates and project coordination, optimised parental experience of care and increased public awareness are some of the potential advantages. Implementing patient involvement is key in making research more meaningful and in developing successful translational research.

\section{PS-122 THE EFFECTS OF ECONOMIC DOWNTURNS ON CHILD MORTALITY: A GLOBAL ANALYSIS, 1981-2010}

${ }^{1} \mathrm{M}$ Maruthappu, ${ }^{2} \mathrm{KYB}$ Ng, ${ }^{3} \mathrm{C}$ Williams, ${ }^{3} \mathrm{E}$ Maclachlan, ${ }^{4} \mathrm{~T}$ Zeltner, ${ }^{5} \mathrm{R}$ Atun. ${ }^{1} \mathrm{Kennedy}$ Scholar, Harvard University, MA, USA; ${ }^{2}$ Paediatrics, Ealing Hospital NHS Trust, London, UK; ${ }^{3}$ Marjorie Deane Intern, The Economist, London, UK; ${ }^{4}$ WHO, World Health Organization, Geneva, Switzerland; ${ }^{5}$ Harvard School of Public Health, Harvard University, MA, USA

\subsection{6/archdischild-2014-307384.417}

Background The effects of economic downturns on population health in high-income countries have been well studied, but less so in low- and middle-income countries. We analysed how 